\title{
Comparison of Natural and BNT162b2 Vaccine- induced Immunity, with and without an Enhancer or Booster Dose, on the Risk of COVID-19-Related Hospitalization in Israel
}

Jacob Waxman ( $\square$ jacobwa@clalit.org.il )

Clalit Research Institute

Maya Makov-Assif

Clalit Research Institute

Ben Reis

Boston Children's Hospital

Doron Nezer

Clalit Health Service

Ran Balicer

Clalit Research Institute https://orcid.org/0000-0002-7783-6362

Noa Dagan

Clalit Research Institute \& Department of Biomedical Informatics, Harvard Medical School

Noam Barda

Clalit Research Institute

\section{Brief Communication}

Keywords:

Posted Date: January 11th, 2022

DOI: https://doi.org/10.21203/rs.3.rs-1177969/v1

License: (c) (1) This work is licensed under a Creative Commons Attribution 4.0 International License. Read Full License

Version of Record: A version of this preprint was published at Nature Communications on April 22nd, 2022. See the published version at https://doi.org/10.1038/s41467-022-29858-5. 


\section{Abstract}

\section{Introduction:}

With the COVID-19 pandemic ongoing, accurate assessment of population immunity and the effectiveness of booster and enhancer vaccines is critical.

\section{Methods}

We compare COVID-19-related hospitalization incidence rate ratios, adjusted for potential demographic, clinical and health-seeking-behavior confounders, in 2,412,755 individuals (235,552,274 person-days), across four exposures: 2 BNT162b2 doses, 5 or more months prior ("non-recent vaccine immunity"); 3 BNT162b2 doses ("boosted vaccine immunity"); previous COVID-19, with or without a subsequent BNT162b2 dose ("natural immunity" and "enhanced natural immunity" respectively).

\section{Results}

Compared with non-recent vaccine immunity, COVID-19-related hospitalization incidence rate ratios are $11 \%(9 \%-13 \%)$ for boosted vaccine immunity, $34 \%$ (23\%-50\%) for natural immunity and $25 \%(17 \%-39 \%)$ for enhanced natural immunity.

\section{Conclusion}

We demonstrate that natural immunity (enhanced or not) provides better protection against COVID-19related hospitalization than non-recent vaccine immunity, but less protection than that attained from booster vaccination. Additionally, our results suggest that vaccinating individuals with natural immunity further enhances their protection.

\section{Introduction}

Nearly two years after the first reported case of SARS-CoV-2, the COVID-19 pandemic is still ongoing, with many countries experiencing new waves of infections and entering further lockdowns. Widespread vaccination campaigns are underway all over the world, although with extremely variable levels of population coverage (1). Surges of healthcare utilization caused by pandemic wave peaks still pose a challenge to the capacity of healthcare systems.

Evidence of waning vaccine immunity over time has emerged: following the second vaccine, there is a significant drop in effectiveness against symptomatic infection; from a peak of approximately $90 \%$ in the weeks immediately following vaccination to a much lower $50 \%-80 \% 6$ months after vaccination (2-6). As a result, some countries are offering booster vaccinations, amongst them Israel, in which over 4 
million individuals received a BNT162b2 booster dose (7). Studies from these countries have now demonstrated the benefit of booster vaccines in reducing symptomatic COVID-19 infection and an even greater reduction in severe outcomes $(8,9)$.

In contrast to this, the degree of protection provided by previous SARS-CoV-2 infection, also known as natural immunity, remains unclear. Recent reports demonstrate what appears to be robust protection from natural immunity against documented reinfection, however, both positive SARS-CoV-2 polymerase chain reaction (PCR) test and symptomatic COVID-19 as outcomes are extremely sensitive to misclassification bias due to differential testing rates, as alluded to by the authors of these reports $(10,11)$. The degree of protection against outcomes that are less vulnerable to bias, such as COVID-19-related hospitalization, remains unclear. Policies regarding the management of recovered COVID-19 patients vary between countries, with some, including Israel, opting to vaccinate these individuals with a single 'enhancer' dose 3 months after diagnosis of the infection (12). This policy is supported by the limited evidence that does exist, which demonstrates that recovered COVID-19 patients who receive a single vaccine dose have an approximately 2 -fold reduced rate of reinfection compared to similar unvaccinated recovered individuals $(11,13)$.

As public health officials face continuing waves of infection, it is critical to understand how vaccineinduced immunity (with and without a booster vaccine) compares with natural immunity (with and without an enhancer vaccine) in preventing COVID-19-related hospitalization. In this retrospective cohort study, we aim to shed light on this question by comparing four groups: Individuals with "non-recent vaccine immunity" (defined as individuals with no prior documented SARS-CoV-2 infection and with at least 5 months following their second BNT162b2 dose, but no third 'booster' dose), "boosted vaccine immunity" (individuals with no prior documented SARS-CoV-2 infection and at least one week following their third BNT162b2 'booster' dose), "natural immunity" (at least 3 months following documented SARSCoV-2 infection and no prior vaccination), and "enhanced natural immunity" (natural immunity, but at least 7 days after a single BNT162b2 'enhancer' dose).

\section{Methods}

\section{Data}

We analyzed observational data from Clalit Health Services (CHS), a nationwide healthcare organization which insures over 4.7 million individuals, over half of the Israeli population. The CHS-covered population is approximately representative of the general Israeli population. CHS provides all outpatient and some inpatient care to its members. CHS began to use electronic medical records over 20 years ago, with data stored centrally. All national COVID-19 data, including PCR test results, diagnoses, severity classification, and vaccinations, are collected centrally by the Israeli Ministry of Health and shared, daily, with the four national health care organizations, including CHS.

\section{Study Design and Population}


This retrospective cohort study analyzed data from July 30, 2021 through November 30, 2021, coinciding with the period of the booster vaccine campaign in Israel, when the Delta variant was dominant. We compared four exposures: "non-recent vaccine immunity" (SARS-CoV-2-naïve individuals with two doses of BNT162b2 and at least 5 months following the second dose and therefore eligible for a booster dose), "boosted vaccine immunity" (SARS-CoV-2-naïve individuals with three doses of BNT162b2, the third dose at least 7 days previously), "natural immunity" (at least 3 months following documented positive SARSCoV-2 PCR test, and no prior or subsequent vaccination) and "enhanced natural immunity" (at least 7 days from single enhancer dose of BNT162b2 given at least 3 months following documented positive SARS-CoV-2 PCR test). The outcome of interest was COVID-19-related hospitalization as determined by the treating physician in each hospital. The use of a 3 month window for defining SARS-CoV-2 reinfection is in line with the Centers for Disease Control and Prevention (CDC) definition (14).

Baseline eligibility criteria were assessed on July 30, 2021 and further eligibility criteria were assessed daily throughout the study period, with individuals only contributing time to follow-up upon meeting both baseline and additional critieria. Baseline eligibility included: age 16 years or older, and continuous membership in the healthcare organization for at least one year. In a similar manner to previous studies $(15,16)$, we excluded individuals belonging to populations in which confounding could not be adequately addressed - specifically healthcare workers, residents of long-term care facilities, house-bound individuals, and individuals for whom data on body-mass index, sector or residential area were missing (rare in the CHS data). Specifically for the two natural immunity groups, only individuals for whom at least 3 months had passed since their SARS-CoV-2 infection on July 30, 2021, were included in order to ensure that the original infection in the natural immunity exposure groups was not from the Delta variant, which came to prevalence in Israel during June 2021 (17).

Individuals meeting the above eligibility criteria were included dynamically in the study population during any day of the study period in which they met the exposure definitions (for example, once 5 months had elapsed from their second dose). Exposure was determined in a time-varying manner, such that individuals were able to contribute time to multiple exposure groups. For example, an individual could contribute time as "non-recent vaccine immunity" until the date the individual received a booster vaccine, and as "boosted vaccine immunity" from 7 days following the booster vaccine. Likewise with natural immunity and enhanced natural immunity. In addition, we excluded pregnant women or women in the postpartum period ( 6 weeks following birth) from the relevant period of follow-up.

In order to ensure that all individuals included in the analysis could receive all exposure levels, individuals were considered 'under analysis' only once their age-group was eligible to receive the booster dose. The booster vaccine campaign in Israel was rolled out in a stepwise manner, with booster vaccines being offered to increasingly younger age-groups as follows: Individuals aged 60 and older from July 30, 2021; individuals aged 50 to 59 from August 12, 2021; individuals aged 40 to 49 from August 19, 2021; individuals aged 30 to 39 from August 24, 2021; and individuals aged 16 to 29 from August 30, 2021. (Hence, for example, individuals aged 50 to 59, were only eligible to start follow-up from August 12, 2021.) 
We adjusted for a wide variety of potential confounders, determined by domain expertise to be associated with both the exposure (type of immunity) and the outcome (COVID-19-related hospitalization). These variables included age (as a continuous variable), sex (male or female), week number of follow up (as a continuous variable), type of residency (a six-level categorization including large city, small city, town, village and kibbutz [a type of communal village specific to Israel]), COVID-19 burden by location of residency (defined as the percentage of total tests in the previous week that were positive in a specific residency code), population sector (general Jewish, ultra-orthodox Jewish or Arab), socioeconomic status (a 20-point scale treated as a continuous variable), the number of preexisting chronic conditions (those considered to be risk factors for severe COVID-19 by the Centers for Disease Control and Prevention as of December 20, 2020, divided into seven categories; 0, 1, 2, 3, 4, 5 or >= 6), and, as indicators of healthseeking behavior, number of diagnoses documented in the outpatient setting in the previous year (percentile for age group in 10 year bins) and the number of flu vaccinations in the 5 years prior to the follow-up (as a binary variable). A complete definition of the study variables, including exposures, the outcome and covariates, is included in Supplementary Table S3.

End of follow up for each person was defined as the earliest of the following outcomes: End of the study period, death, termination of healthcare organization membership, or occurrence of the outcome (COVID19-related hospitalization).

\section{Statistical Analysis}

The effect estimate of interest was the Incidence Rate Ratio (IRR). The IRR was estimated using multivariable Poisson regression, with "non-recent vaccine immunity" used as the baseline. Continuous variables (age, week of follow-up, COVID-19 burden by location of residency and socioeconomic status) were modelled as restricted cubic splines with 2, 3, 3 and 3 degrees of freedom, respectively.

The unit of time in the analysis was a single person-day. To allow for time-varying covariates, including a time-varying exposure (e.g., individuals receiving the booster dose during the study period), each person was represented in the data by multiple rows, each with its own covariate values, with a variable representing the number of days "at-risk" in each row used as the "offset" in the model. That means that each individual could contribute time to more than one exposure group.

We opted to perform complete case analysis given that missing data in the variables used is rare in the CHS dataset.

\section{Sensitivity Analysis}

In addition, we performed a sensitivity analysis in which an identical analysis to that described above was performed, but follow-up was limited to a maximum of 5 months from the date of the enhancer vaccine (recent vaccination) in the enhanced natural immunity group, in an attempt to eliminate the effect of waning immunity.

\section{Ethics}


CHS institutional review board approved this study and it was deemed exempt from the requirement for informed consent.

\section{Results}

Of a total of 3,199,145 individuals considered for the analysis, 2,412,755 (75.4\%) were found eligible to enter the analysis. A study population flow chart appears in Figure S1. Median age of the study population was 47 (IQR 33-65) and 51\% were female. The total time under follow-up was 235,552,274 person-days. Only $1 \%$ of cases had missing data. The number of person-days contributed varied between the groups due to differences in the number of individuals contributing to each group and the potential length of time contributed, with the most person-days contributed to boosted vaccine immunity $(143,612,328)$, followed by non-recent vaccine immunity $(72,914,787)$, natural immunity $(9,759,128)$, and enhanced natural immunity $(9,266,031)$. A description of the study population, stratified by exposure groups, is included in Table 1.

Compared with individuals with non-recent vaccine immunity, we estimate that the rate of COVID-19related hospitalization is $11 \%(95 \% \mathrm{Cl} 9 \%-13 \%)$ in individuals with boosted vaccine immunity, 34\% (95\% $\mathrm{Cl} 23 \%-50 \%)$ in individuals with natural immunity and $25 \%$ (95\% $\mathrm{Cl} 17 \%-39 \%)$ in individuals with enhanced natural immunity (Table S1, Figure 1).

We performed a sensitivity analysis, in which the enhanced natural immunity group included only those who received an enhancer vaccine dose less than 5 months ago (recent enhanced natural immunity). The rate of COVID-19-related hospitalization was 25\% (95\% Cl 13\%-48\%) (Table S2) compared with non-recent vaccine immunity.

\section{Discussion}

In this retrospective cohort study of over two million individuals, we demonstrate that receipt of a booster (third) dose of the BNT162b2 SARS-CoV-2 greatly improves protection against COVID-19 related hospitalization. The case for universal booster vaccines remains a contentious issue (18) despite a number of recent publications demonstrating a substantial risk reduction in COVID-19-related outcomes from booster vaccines $(8,9)$. We corroborate previous publications, showing that individuals with nonrecent vaccine immunity benefit from a booster dose, with much increased protection against COVID-19related hospitalization (incidence rate 11\%). Furthermore, the magnitude of our estimated effect size for the benefit of a booster vaccine is in line with previous studies $(8,9)$.

Evidence supporting the use of enhancer doses in recovered SARS-CoV-2 patients exists, but is sparse $(11,13)$. In our study, we found that individuals with natural immunity benefit from an enhancer dose. The two studies to date comparing individuals with natural immunity and enhanced natural immunity on COVID-19-related hospitalization have very small sample sizes. In one of the studies, most individuals had received two vaccine doses following infection (as per the CDC recommendations) and only a small 
number had received only one enhancer dose. In that study a 1.5-fold reduction in the odds ratio was reported for a single enhancer dose and a 2.3-fold reduction for two vaccine doses. The estimated reduced IRR in this study (25\% with an enhancer dose compared with $34 \%$ without an enhancer dose) are consistent with the current evidence.

Perhaps the most noteworthy finding of the present study concerns the relationship between vaccineinduced immunity and natural immunity, with and without booster/enhancer doses. We estimate that natural immunity (with or without an enhancer dose) provides superior protection against COVID-19related hospitalization compared to non-recent vaccine immunity (incidence rate $34 \%$ and $25 \%$, respectively). This confirms findings alluded to in two recent pre-print articles, albeit with low certainty due to small sample size (11) and presentation of unadjusted rates limited to individuals over 60 years old (10).

However, our analysis goes further to demonstrate that the picture dramatically changes with introduction of a booster dose; individuals with boosted vaccine immunity (the majority of vaccinated individuals in Israel) have even higher levels of protection against COVID-19 (incidence rate 11\%) than individuals with natural immunity and enhanced natural immunity (incidence rate $34 \%$ and $25 \%$ respectively). To date, other than the pre-print article, with its limitations mentioned above (10), evidence is lacking as to the relative protection afforded by boosted vaccine immunity and natural immunity (with or without an enhancer dose) against COVID-19-related hospitalization.

An important design consideration in the present study is the choice of COVID-19 related hospitalization as the outcome of interest. This outcome was chosen because it is less prone to bias than positive PCR tests or symptomatic COVID-19 since, to a large extent, it circumvents misclassification arising from differential testing rates across exposure groups. That is, it is less likely for someone requiring hospitalization to be 'missed' because they opted not to get a SARS-CoV-2 PCR test. On the other hand, using more severe and less common outcomes (such as COVID-19-related death) results in less statistical power for estimation. We feel that the chosen outcome represents the best compromise between minimizing bias and maximizing statistical power.

Our study is subject to a number of limitations. First, as in any observational study, and despite the attempts for rigorously controlling for potential confounders, there is still the possibility of residual confounding. Second, we are focusing on the first three months following booster vaccination - likely the period with peak immunity. It is possible that booster immunity wanes over time and further studies will be required to assess the protection from booster vaccination over the longer term. Furthermore, we compare individuals with peak immunity from a recent booster vaccination to individuals with natural immunity possibly attained over a year previously or enhanced natural immunity up to 8 months following the enhancer dose (Figure S2). It could be argued that this is an unfair comparison and that we should instead compare recently boosted individuals to individuals who were recently infected or recently received an enhancer dose. In order to assess the validity of our study design, we performed a sensitivity analysis in which the enhanced natural immunity group was limited to only include follow up time within 
5 months of the enhancer dose (recently enhanced natural immunity) and repeated our analysis. The result, an identical point estimate of $25 \%$ compared with non-recent vaccine immunity, with a wider confidence interval (13\% - 48\%) reflecting the reduced sample size, supports our assertion that the results presented do not simply represent waning of the natural immunity groups (Table S2). Lastly, it must be remembered that the specific exposures that we assessed in this study are vaccination with BNT162b2 and infection with non-Delta variants of SARS-CoV-2 and the specific outcome is hospitalization due to infection with the Delta variant. The results may not be generalizable to different setings and different vaccines, and specifically not when the original infection and the outcome are both from the same variant (e.g. Delta).

In conclusion, we demonstrate that, while natural immunity (with or without an enhancer dose of BNT162b2) provides more protection against COVID-19-related hospitalization than non-recent vaccine immunity, booster vaccination provides an even greater level of relative protection. Furthermore, we also demonstrate that individuals with natural immunity benefit from receipt of an enhancer dose of BNT162b2, although with a smaller relative benefit than that of the booster vaccine in the non-recent vaccine immunity group. We believe these findings can help inform members of the public and decisionmakers around the world, both strengthening their conviction in the benefit and necessity of booster vaccination campaigns and enabling them to assess, more accurately, levels of COVID-19 immunity in the population.

\section{Declarations}

\section{Acknowledgments}

We thank Tal Hollander, Yatir Ben-Shlomo and Shay Perchick for their advice on analysis and Jonathan Assif and Jonathan Waxman on their advice on the write-up.

\section{Funding}

None.

\section{Author contributions}

J.G.W. and M.M.A. contributed equally as first authors. N.D. and N.B. contributed equally as senior authors to this study. J.G.W., M.M.A., N.D., N.B., and R.D.B. conceived and designed the study. J.G.W., M.M.A., N.D., and N.B. participated in data extraction and analysis. J.G.W., M.M.A., B.Y.R., D.N., N.D., and N.B. wrote the manuscript. D.N. provided clinical guidance. All authors critically reviewed the manuscript and decided to proceed with publication. N.D., and N.B. supervised the study and vouch for the data and analysis. 


\section{Disclosure of interest}

J.G.W, N.D., N.B., M.M.A. and R.D.B. report institutional grants to the Clalit Research Institute from Pfizer outside the submitted work and unrelated to COVID-19, with no direct or indirect personal benefits. BYR reports grants from $\mathrm{NIH}$ outside the submitted work.

\section{Data availability statement}

Due to national and organizational data privacy regulations, individual-level data such as those used for this study cannot be shared openly.

\section{Code availability}

The modeling in this paper used R v.4.1.0 and the tidyverse v.1.3.1 R packages, all of which are freely available.

\section{References}

1. Coronavirus (COVID-19) Vaccinations - Statistics and Research - Our World in Data [Internet]. [cited 2021 Nov 28]. Available from: https://ourworldindata.org/covid-vaccinations

2. Chemaitelly H, Tang P, Hasan MR, AlMukdad S, Yassine HM, Benslimane FM, et al. Waning of BNT162b2 Vaccine Protection against SARS-CoV-2 Infection in Qatar. N Engl J Med. 2021 Oct 6;

3. Levin EG, Lustig Y, Cohen C, Fluss R, Indenbaum V, Amit S, et al. Waning Immune Humoral Response to BNT162b2 Covid-19 Vaccine over 6 Months. N Engl J Med. 2021 Oct 6;

4. Andrews N, Tessier E, Stowe J, Gower C, Kirsebom F, Simmons R, et al. Vaccine effectiveness and duration of protection of Comirnaty, Vaxzevria and Spikevax against mild and severe COVID-19 in the UK. medRxiv. 2021 Sep 21;

5. Goldberg Y, Mandel M, Bar-On YM, Bodenheimer O, Freedman L, Haas EJ, et al. Waning immunity of the BNT162b2 vaccine: A nationwide study from Israel. medRxiv. 2021 Aug 30;

6. Thomas SJ, Moreira ED, Kitchin N, Absalon J, Gurtman A, Lockhart S, et al. Six Month Safety and Efficacy of the BNT162b2 mRNA COVID-19 Vaccine. medRxiv. 2021 Jul 28;

7. COVID-19 vaccine booster doses administered per 100 people - Our World in Data [Internet]. [cited 2021 Nov 29]. Available from: https://ourworldindata.org/grapher/covid-vaccine-booster-doses-percapita?country=BRA CHL ISR RUS USA URY OWID_WRL 
8. Barda N, Dagan N, Cohen C, Hernán MA, Lipsitch M, Kohane IS, et al. Effectiveness of a third dose of the BNT162b2 mRNA COVID-19 vaccine for preventing severe outcomes in Israel: an observational study. Lancet. 2021 Oct 29;

9. Bar-On YM, Goldberg Y, Mandel M, Bodenheimer O, Freedman L, Kalkstein N, et al. Protection of BNT162b2 Vaccine Booster against Covid-19 in Israel. N Engl J Med. 2021 Oct 7;385(15):1393-1400.

10. $\mathrm{MOH}$. Israel Ministry of Health Guidelines on Vaccinating Recovered Covid-19 Patients [Internet]. 2021 [cited 2021 Nov 28]. Available from: https://www.gov.il/en/departments/news/01032021-02

1. Coronavirus (COVID-19) Vaccinations - Statistics and Research - Our World in Data [Internet]. [cited 2021 Nov 28]. Available from: https://ourworldindata.org/covid-vaccinations

2. Chemaitelly H, Tang P, Hasan MR, AlMukdad S, Yassine HM, Benslimane FM, et al. Waning of BNT162b2 Vaccine Protection against SARS-CoV-2 Infection in Qatar. N Engl J Med. 2021 Oct 6;

3. Levin EG, Lustig Y, Cohen C, Fluss R, Indenbaum V, Amit S, et al. Waning Immune Humoral Response to BNT162b2 Covid-19 Vaccine over 6 Months. N Engl J Med. 2021 Oct 6;

4. Andrews N, Tessier E, Stowe J, Gower C, Kirsebom F, Simmons R, et al. Vaccine effectiveness and duration of protection of Comirnaty, Vaxzevria and Spikevax against mild and severe COVID-19 in the UK. medRxiv. 2021 Sep 21;

5. Goldberg Y, Mandel M, Bar-On YM, Bodenheimer O, Freedman L, Haas EJ, et al. Waning immunity of the BNT162b2 vaccine: A nationwide study from Israel. medRxiv. 2021 Aug 30;

6. Thomas SJ, Moreira ED, Kitchin N, Absalon J, Gurtman A, Lockhart S, et al. Six Month Safety and Efficacy of the BNT162b2 mRNA COVID-19 Vaccine. medRxiv. 2021 Jul 28;

7. COVID-19 vaccine booster doses administered per 100 people - Our World in Data [Internet]. [cited 2021 Nov 29]. Available from: https://ourworldindata.org/grapher/covid-vaccine-booster-doses-percapita? country=BRA CHL ISR RUS USA URY OWID_WRL

8. Barda N, Dagan N, Cohen C, Hernán MA, Lipsitch M, Kohane IS, et al. Effectiveness of a third dose of the BNT162b2 mRNA COVID-19 vaccine for preventing severe outcomes in Israel: an observational study. Lancet. 2021 Oct 29;

9. Bar-On YM, Goldberg Y, Mandel M, Bodenheimer O, Freedman L, Kalkstein N, et al. Protection of BNT162b2 Vaccine Booster against Covid-19 in Israel. N Engl J Med. 2021 Oct 7;385(15):1393-1400.

10. Goldberg Y, Mandel M, Bar-On YM, Bodenheimer O, Freedman L, Alroy-Preis S, et al. Protection and waning of natural and hybrid COVID-19 immunity. medRxiv. 2021 Dec 5; 
11. Gazit S, Shlezinger R, Perez G, Lotan R, Peretz A, Ben-Tov A, et al. Comparing SARS-CoV-2 natural immunity to vaccine-induced immunity: reinfections versus breakthrough infections. medRxiv. 2021 Aug 25;

12. $\mathrm{MOH}$. Israel Ministry of Health Guidelines on Vaccinating Recovered Covid-19 Patients [Internet]. 2021 [cited 2021 Nov 28]. Available from: https://www.gov.il/en/departments/news/01032021-02

13. Cavanaugh AM, Spicer KB, Thoroughman D, Glick C, Winter K. Reduced Risk of Reinfection with SARS-CoV-2 After COVID-19 Vaccination - Kentucky, May-June 2021. MMWR Morb Mortal Wkly Rep. 2021 Aug 13;70(32):1081-1083.

14. Ending Isolation and Precautions for People with COVID-19: Interim Guidance [Internet]. [cited 2021 Dec 4]. Available from: https://www.cdc.gov/coronavirus/2019-ncov/hcp/duration-isolation.html

15. Barda N, Dagan N, Ben-Shlomo Y, Kepten E, Waxman J, Ohana R, et al. Safety of the BNT162b2 mRNA Covid-19 Vaccine in a Nationwide Setting. N Engl J Med. 2021 Sep 16;385(12):1078-1090.

16. Dagan N, Barda N, Kepten E, Miron O, Perchik S, Katz MA, et al. BNT162b2 mRNA Covid-19 Vaccine in a Nationwide Mass Vaccination Setting. N Engl J Med. 2021 Apr 15;384(15):1412-1423.

17. SARS-CoV-2 variants in analyzed sequences - Our World in Data [Internet]. [cited 2021 Dec 7]. Available from: https://ourworldindata.org/grapher/covid-variants-area?country= ISR

18. Scott J, Richterman A, Cevik M. Covid-19 vaccination: evidence of waning immunity is overstated. BMJ. 2021 Sep 23;374:n2320.

\section{Tables}

Table 1 - Baseline characteristics of study population following application of all eligibility criteria 


\begin{tabular}{|c|c|c|c|c|}
\hline Characteristic & $\begin{array}{l}\text { Non-recent } \\
\text { Vaccine } \\
\text { immunity, } \\
\mathrm{N}= \\
2,169,543^{*} \\
(72,914,787 \\
\text { person-days } \\
\text { at risk) }\end{array}$ & $\begin{array}{l}\text { Boosted vaccine } \\
\text { immunity, } \\
N=1,739,990^{*} \\
(143,612,328 \\
\text { person-days at } \\
\text { risk) }\end{array}$ & $\begin{array}{l}\text { Natural } \\
\text { immunity, N } \\
=126,3771^{*} \\
(9,759,128 \\
\text { person-days } \\
\text { at risk) }\end{array}$ & $\begin{array}{l}\text { Enhanced natural } \\
\text { immunity, } \\
\mathrm{N}=125,6251 * \\
(9,266,031 \\
\text { person-days at } \\
\text { risk) }\end{array}$ \\
\hline Age & $46(32,64)$ & $49(35,67)$ & $34(24,47)$ & $38(26,52)$ \\
\hline \multicolumn{5}{|l|}{ Sex } \\
\hline Female & $\begin{array}{l}1,103,228 \\
(51 \%)\end{array}$ & $885,688(51 \%)$ & $68,176(54 \%)$ & 68,549 (55\%) \\
\hline Male & $\begin{array}{l}1,066,315 \\
(49 \%)\end{array}$ & $854,302(49 \%)$ & $58,201(46 \%)$ & $57,076(45 \%)$ \\
\hline \multicolumn{5}{|l|}{ Sector } \\
\hline General Jewish & $\begin{array}{l}1,604,473 \\
(74 \%)\end{array}$ & $1,396,001(80 \%)$ & $64,290(51 \%)$ & 67,958 (54\%) \\
\hline Ultra-orthodox & $\begin{array}{l}70,871 \\
(3.3 \%)\end{array}$ & $53,231(3.1 \%)$ & 19,948 (16\%) & 13,607 (11\%) \\
\hline Arab & $\begin{array}{l}494,199 \\
(23 \%)\end{array}$ & $290,758(17 \%)$ & $42,139(33 \%)$ & $44,060(35 \%)$ \\
\hline \multicolumn{5}{|l|}{ CDC risk factors } \\
\hline 0 & $\begin{array}{l}1,066,576 \\
(49 \%)\end{array}$ & $807,761(46 \%)$ & $75,151(59 \%)$ & 68,987 (55\%) \\
\hline 1 & $\begin{array}{l}532,955 \\
(25 \%)\end{array}$ & $429,798(25 \%)$ & $31,396(25 \%)$ & 31,735 (25\%) \\
\hline 2 & $\begin{array}{l}262,200 \\
(12 \%)\end{array}$ & $226,325(13 \%)$ & $10,473(8.3 \%)$ & $12,699(10 \%)$ \\
\hline 3 & $\begin{array}{l}155,463 \\
(7.2 \%)\end{array}$ & $138,738(8.0 \%)$ & $4,745(3.8 \%)$ & $6,338(5.0 \%)$ \\
\hline 4 & $\begin{array}{l}86,772 \\
(4.0 \%) \\
\end{array}$ & 78,285 (4.5\%) & $2,547(2.0 \%)$ & $3,319(2.6 \%)$ \\
\hline 5 & $\begin{array}{l}41,851 \\
(1.9 \%) \\
\end{array}$ & $37,761(2.2 \%)$ & $1,227(1.0 \%)$ & $1,574(1.3 \%)$ \\
\hline 6 & $\begin{array}{l}23,726 \\
(1.1 \%) \\
\end{array}$ & $21,322(1.2 \%)$ & $838(0.7 \%)$ & $973(0.8 \%)$ \\
\hline \multicolumn{5}{|l|}{ Residency type } \\
\hline Large city & $\begin{array}{l}822,888 \\
(38 \%)\end{array}$ & $681,510(39 \%)$ & 46,877 (37\%) & $44,286(35 \%)$ \\
\hline Small city & $\begin{array}{l}778,757 \\
(36 \%) \\
\end{array}$ & $606,594(35 \%)$ & $50,071(40 \%)$ & $48,333(38 \%)$ \\
\hline Town & $\begin{array}{l}329,110 \\
(15 \%)\end{array}$ & $241,092(14 \%)$ & $22,652(18 \%)$ & 24,427 (19\%) \\
\hline Village & $\begin{array}{l}156,215 \\
(7.2 \%) \\
\end{array}$ & $133,623(7.7 \%)$ & $5,942(4.7 \%)$ & $6,779(5.4 \%)$ \\
\hline Kibbutz & 82,573 & $77,171(4.4 \%)$ & $835(0.7 \%)$ & $1,800(1.4 \%)$ \\
\hline
\end{tabular}




\begin{tabular}{|l|l|l|l|l|} 
& $(3.8 \%)$ & & \\
\hline $\begin{array}{l}\text { Flu vaccines in } \\
\text { the past 5 years }\end{array}$ & $\begin{array}{l}1,048,397 \\
(48 \%)\end{array}$ & $932,457(54 \%)$ & $33,779(27 \%)$ & $47,147(38 \%)$ \\
\hline $\begin{array}{l}\text { Number of } \\
\text { diagnoses } \\
\text { recorded in } \\
\text { outpatient setting } \\
\text { (age-adjusted } \\
\text { percentile) }\end{array}$ & $\begin{array}{l}0.45(0.20, \\
0.73)\end{array}$ & $0.48(0.22,0.73)$ & $\begin{array}{l}0.62(0.41, \\
0.82)\end{array}$ & $0.66(0.45,0.84)$ \\
\hline $\begin{array}{l}\text { Length of follow } \\
\text { up in Days }\end{array}$ & $18(7,48)$ & $89(64,102)$ & $94(44,100)$ & $87(45,100)$ \\
\hline
\end{tabular}

${ }^{1}$ Median (IQR); n (\%)

* Individuals can appear in more than one column if their exposure changed during the study

\section{Figures}

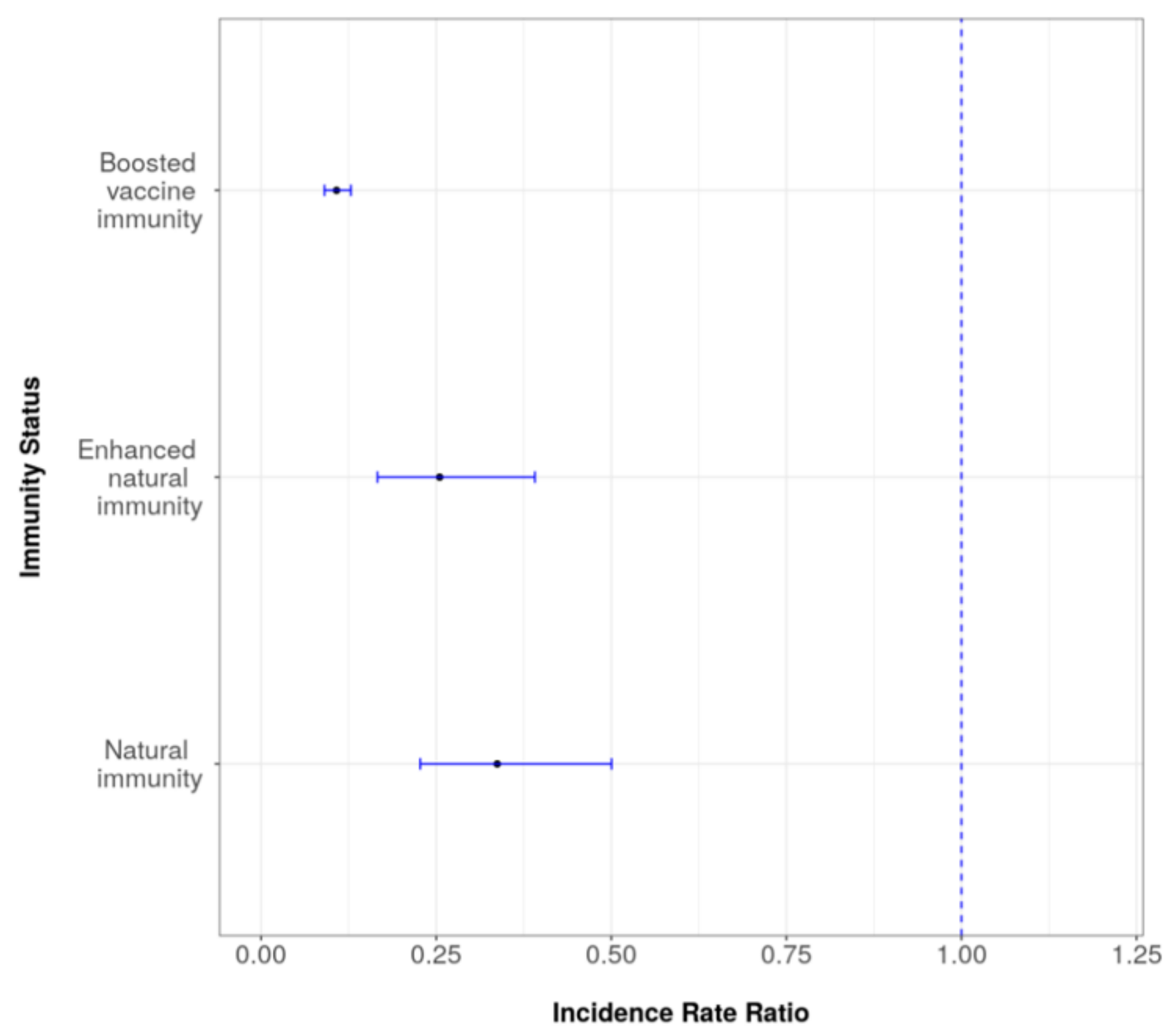

Page $13 / 14$ 
Figure 1

A graph showing the incidence rate ratio of COVID-19-related hospitalization amongst individuals with boosted vaccine immunity, natural immunity and enhanced natural immunity compared with individuals with non-recent vaccine immunity (the dotted line at 1.00 )

\section{Supplementary Files}

This is a list of supplementary files associated with this preprint. Click to download.

- SupplementaryMaterials.docx 